\section{G. Wagner ${ }^{1}$ \\ C. Diaz $^{2}$ \\ W. Weyers ${ }^{2}$}

\title{
Lokalisierter Morbus Darier
}

\section{Localized Darier's Disease}

\section{Zusammenfassung}

Aufgrund klinischer und histopathologischer Befunde wurde bei dem hier vorgestellten Patienten die Diagnose eines lokalisierten Morbus Darier gestellt. Der lokalisierte Morbus Darier ist wahrscheinlich Folge einer postzygoten Mutation, die zu einer genetischen Mosaikbildung führt. Von Ausnahmen abgesehen, zeigen die lokalisierten Formen des Morbus Darier keine weiteren klinischen Merkmale der klassischen Form der Dermatose. Vor dem Hintergrund der von Happle formulierten Definition des Nävus als Folge eines genetischen Mosaiks ist die Frage, ob es sich bei den lokalisierten Formen des Morbus Darier um eine Variante der Dermatose oder um einen Nävus im Sinne eines akantholytischen dyskeratotischen epidermalen Nävus handelt, hinfällig, da beide Auffassungen miteinander vereinbar sind.

\section{Abstract}

Diagnosis of localized Darier's Disease was made on the basis of clinical and histological findings. Localized Darier's Disease is probably caused by a postzygotic mutation, leading to a genetic mosaic. Apart from exceptions, localized forms of Darier's Disease do not show further clinical characteristics of classical Darier's Diseace. Considering Happle's definition of nevus as result of a genetic mosaic, the discussion about whether localized Darier's Disease is a variant of the generalized dermatosis, or an acantholytic dyskeratotic epidermal nevus, is out-dated. Both views are consistent.

\section{Einleitung}

Die Dyskeratosis follicularis Darier ist eine autosomal-dominant vererbte Genodermatose mit hoher Penetranz, ausgeprägter Spontanmutationsrate und einer variablen, von exogenen Faktoren beeinflussten Ausprägung der jeweils vorliegenden Symptomatik [1]. Pathogenetisch ist der Morbus Darier durch einen Verlust der epidermalen Zelladhäsion und durch eine gestörte Keratinisierung gekennzeichnet. Der für die Erkrankung verantwortliche genetische Defekt konnte auf dem Chromosom 12 in der Region 12 q23-q24.1 kartiert werden [2]. Sakuntabhai et al. gelang es 1999, verschiedene Mutationen im ATP2A2-Gen der genannten Region nachzuweisen [3]. Das Gen kodiert eine im sarkoendoplasmatischen Retikulum lokalisierte kalziumabhängige ATPase (SERCA2), die für den aktiven Transport von Kalzium-Io- nen vom Zytosol in das Retikulum verantwortlich ist. Die Ausbildung epithelialer Zellkontakte durch Desmosomen und die Differenzierung der Epidermis sind dabei von der zytosolischen Kalzium-Konzentration abhängig. Möglicherweise sind die unterschiedlichen Mutationen für die phänotypischen Variationen des Morbus Darier verantwortlich. Dabei ist das klinische Bild der Dermatose insgesamt charakteristisch. Typischerweise entwickeln sich im ersten oder zweiten Lebensjahrzehnt zunächst linsengroße, graubraun oder dunkelbraun pigmentierte, keratotische Papeln, die einzeln, gruppiert oder auch zu größeren Beeten konfluierend auftreten können. Zu den Prädilektionsstellen, in denen sich die Papeln in symmetrischer Anordnung langsam progredient entwickeln, zählen die seborrhoischen und intertriginösen Areale, das Gesicht und der behaarte Kopf sowie die großen Gelenkbeugen. Palmoplantare Keratosen, punktförmige Un- 
terbrechungen der Papillarlinien sowie Nagelplatten- und Schleimhautveränderungen vervollständigen das klinische Bild [4]. Zu den histopathologischen Befunden des Morbus Darier gehören neben einer Akanthose und Hyperkeratose, eine suprabasale akantholytische Spaltbildung sowie eine fokale Dyskeratose mit Corps ronds, großen dyskeratotischen Zellen, und Grains, parakeratorischen Zellen [5,6].

Im Gegensatz zum klassischen klinischen Bild des Morbus Darier sind vereinzelt lokalisierte Manifestationsformen beobachtet worden. Die verschiedenen Ausbreitungsmuster dieser umschriebenen Formen können im Allgemeinen dem System der Blaschko-Linien zugeordnet werden. Auch der hier vorgestellte Fall ist als lokalisierter Morbus Darier einzuordnen, wobei die umschriebene Manifestation in Form eines aus mehreren aggregierten Papeln bestehenden singulären Herdes als außergewöhnlich anzusehen und mit keinem zweiten Fall in der uns zur Verfügung stehenden Literatur vergleichbar gewesen ist.

\section{Kasuistik}

\section{Anamnese}

Der jetzt 74-jährige Patient hatte vor zwei Jahren erstmals nicht juckende Hautveränderungen am vorderen Dekolleté links bemerkt. Dabei waren zunächst kleinere Herde aufgetreten, die anschließend zu einem größeren Areal konfluierten. Seit etwa einem Jahr hatte sich der Befund in Form und Größe nicht mehr verändert. Die Familienanamnese war unauffällig. Bei den Eltern, Geschwistern, Kindern und Enkelkindern des Patienten ergaben sich insbesondere keine Hinweise für einen bekannt gewordenen Morbus Darier.

\section{Dermatologischer Befund}

Am vorderen Dekolleté links lateral auf einem ca. $4 \mathrm{~cm}$ großen Erythem gruppiert stehende, 2-5 mm durchmessende, teilweise konfluierende Papeln mit verruköser Oberfläche. In der unmittelbaren Umgebung einzelne Papeln gleicher Morphologie. Derber Palpationsbefund (Abb.1 u.2). Übriges Hautorgan unauffällig. Keine pathologischen Befunde im Bereich der Mundschleimhäute oder an den Nagelplatten der Finger. Im Daktylogramm keine Unterbrechungen der Papillarlinien.

\section{Histopathologischer Befund}

Das 4mm-Stanzbiopsat zeigt zwei voneinander getrennte Bereiche mit suprabasaler Akantholyse. Die Epidermis weist hier eine perpendikuläre Proliferation der Basalzellen auf. In höheren Epidermislagen finden sich Dyskeratosen, übergehend in eine verbreiterte, parakeratotische Hornschicht (Abb. 3u.4). Der Befund ist charakteristisch für eine fokale akantholytische Dyskeratose. Dieser Befund ist nicht spezifisch, aber charakteristisch für einen Morbus Darier.

\section{Therapie und Verlauf}

Unter Anwendung von Salizyl-Vaseline 5\% bildeten sich die Hyperkeratosen nur teilweise zurück. Bei einer Wiedervorstellung nach sechs Monaten waren keine neuen Herde aufgetreten.

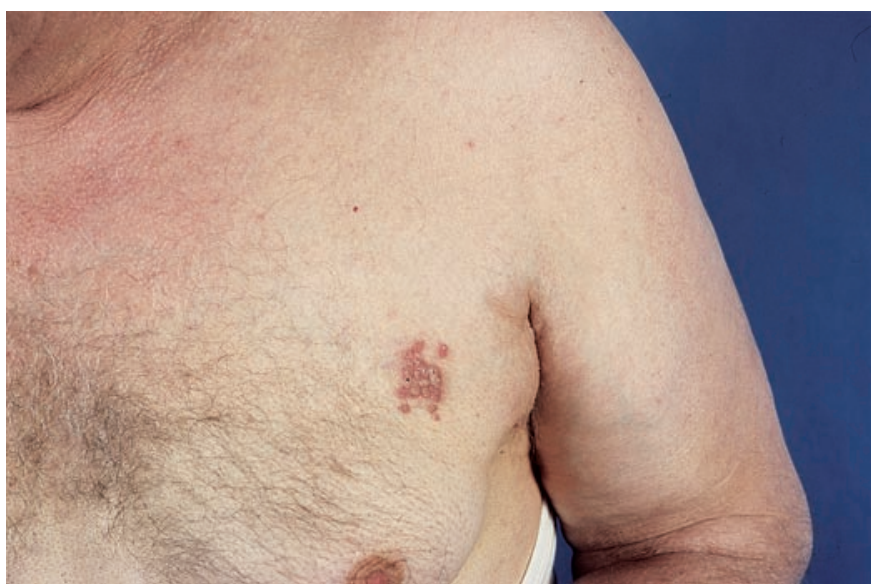

Abb. 1 Lokalisierter Morbus Darier pektoral links.

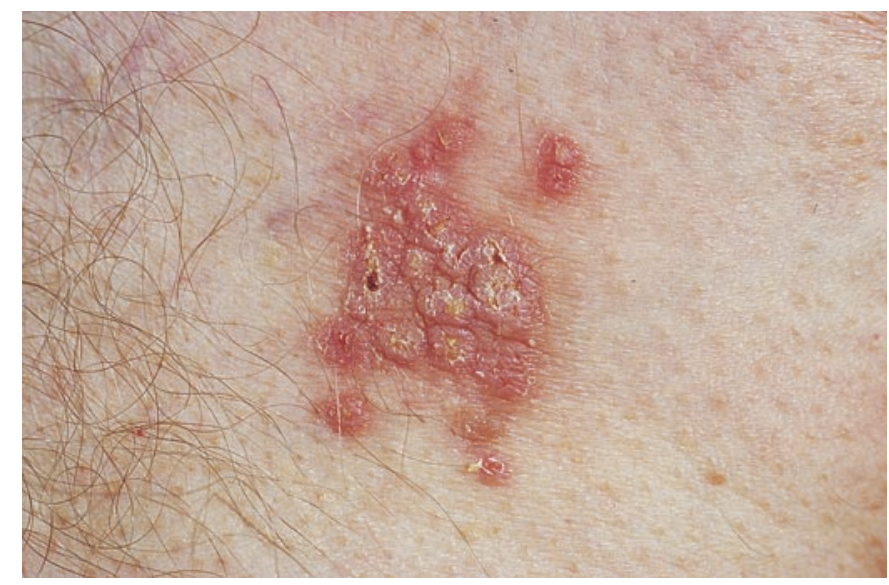

Abb. 2 Detailaufnahme.

\section{Diskussion}

Die in begrenzten Arealen auftretenden Formen des Morbus Darier sind je nach klinischem Ausbreitungsmuster als linear, zosteriform, näviform, segmentär oder unilateral beschrieben worden. [1,7-10]. In der angloamerikanischen Literatur werden diese Manifestationen meist einheitlich als lokalisierte Formen bezeichnet $[11,12]$. Da die im deutschen Sprachgebrauch verwendeten Adjektive der allen umschrieben auftretenden Manifestationsformen gemeinsamen genetischen Störung nicht gerecht werden, erscheint der übergeordnete Begriff der lokalisierten Formen sinnvoller.

Der Morbus Darier gilt als eine seltene Dermatose. Gesicherte epidemiologische Daten finden sich in der Literatur kaum, wobei Prävalenzraten von 1:55000, 1:75000 und 1:100000 angegeben werden [13-15]. Noch seltener sind lokalisierte Manifestationen beobachtet worden. Ihre Häufigkeit wird auf $1-10 \%$ aller Darier-Erkrankungen geschätzt [1].

O'Malley et al. konnten bis 1997 in der englischsprachigen Literatur einschließlich eigener Beobachtungen insgesamt 40 lokalisierte Darier-Fälle zusammenstellen [12]. Innerhalb dieser Gruppe war die Geschlechtsverteilung mit jeweils 20 betroffenen Patientinnen und Patienten ausgeglichen. Das mittlere Alter der 


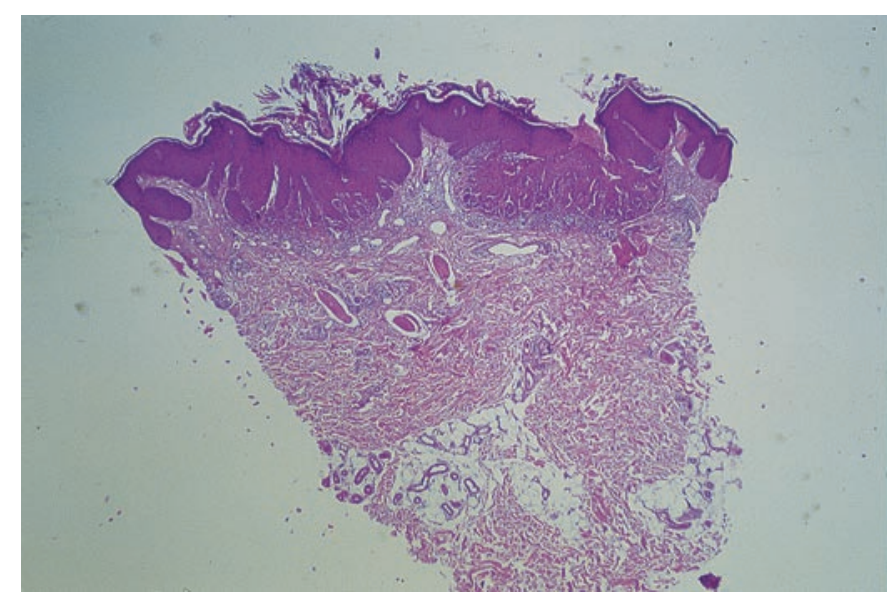

Abb. 3 Die Übersichtsvergrößerung zeigt zwei durch unaufällige Epidermis voneinander getrennte Bereiche mit fokaler akantholytischer Dyskeratose (HE, 1:20).

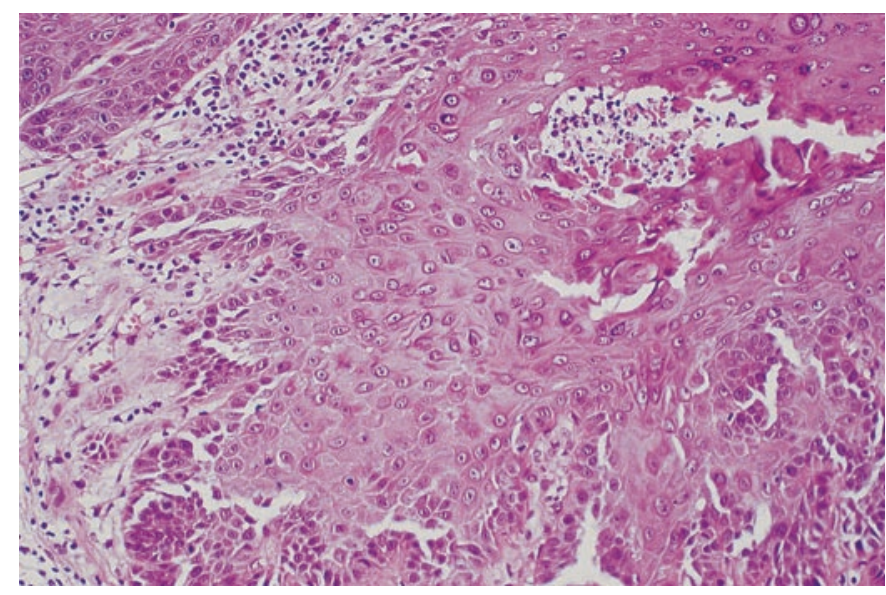

Abb. 4 Zur Tiefe hin weist die Epidermis perpendikuläre Verlängerungen der Basalzellschicht auf. Suprabasal findet sich eine Akantholyse. Im Stratum spinosum einzelne Dyskeratosen. Parakeratorische Hautschicht (HE, 1:250).

Patienten betrug zu Beginn der Dermatose 27 Jahre, wobei 12 der 40 Patienten zu diesem Zeitpunkt älter als 30 Jahre waren. Bei dem jüngsten Patienten lagen die Hautveränderungen bereits bei der Geburt vor, während sich die Dermatose bei dem ältesten Patienten erst mit 79 Jahren manifestierte. Somit treten die lokalisierten Formen des Morbus Darier bei einem Großteil der Patienten erst in einem höheren Lebensalter auf, im Gegensatz zur klassischen Form der Dermatose, die üblicherweise bereits in der Kindheit oder in der Adoleszenz beobachtet wird [4]. Auch die Anordnung und die Verteilung der lokalisierten Formen unterscheiden sich von den Befunden des klassischen Morbus Darier. Die lokalisierten Formen treten solitär oder in wenigen Einzelherden auf, zeigen keine symmetrische Verteilung und finden sich in der Regel nicht im Bereich der intertriginösen oder seborrhoischen Areale. Der lokalisiert auftretende Morbus Darier zeigt sich bevorzugt am Rücken, lateral am Stamm sowie im Bereich der Extremitäten. Nur selten finden sich bei einem lokalisierten Morbus Darier assoziierte Symptome, wie z.B. Pigmentierungen, Keratosen der Handrücken und Schleimhäute, Unterbrechungen der Papillarlinien oder Störungen des Nagelwachstums [1,12]. Des Weiteren fehlt bei den lokalisierten Formen der sonst typi- sche lebenslang progrediente Verlauf des Morbus Darier. Die Häufigkeit, das Manifestationsalter, die Lokalisation der Effloreszenzen, das Fehlen oder der Nachweis assoziierter Symptome und der Verlauf können somit als klinische Kriterien herangezogen werden, um die lokalisierten Formen grundsätzlich von der klassischen Ausprägung des Morbus Darier abzugrenzen. Andererseits gibt es zahlreiche Befunde, die die gemeinsame nosologische Einordnung der verschiedenen Darier-Formen begründen. Zu diesen Merkmalen zählen die charakteristische primäre Effloreszenz der hyperkeratotischen Papel, die einheitlichen Befunde in der Licht- und Elektronenmikroskopie sowie die gemeinsamen exogenen Provokationsfaktoren Wärme, Transpiration und Sonnenlicht $[1,4,12,16]$. Auch das therapeutische Ansprechen auf die gleichen Behandlungsverfahren ist bei allen Formen des Morbus Darier identisch $[4,12,17]$.

Ein weiterer interessanter Aspekt der lokalisierten Darier-Formen ist deren genetische Einordnung. Während der Morbus Darier in seiner klassischen Form zu den autosomal-dominant vererbten Genodermatosen gezählt wird, ist die Stellung der lokalisierten Formen längere Zeit kontrovers diskutiert worden. Zum einen wurden die lokalisierten Formen als Varianten der klassischen Manifestation des Morbus Darier dargestellt, zum anderen als epidermale Nävi eingeordnet. Von Starink und Woerdeman wurde erstmals der Name akantholytischer dyskeratotischer epidermaler Nävus verwendet [17]. Die Beobachtung singulärer Herde oder die bilaterale asymmetrische Verteilung mehrerer Herde, die nicht vorhandene familiäre Belastung und das Fehlen assoziierter Symptome wurden zunächst als Hinweise für eine Nävus-Genese gewertet $[17,18]$. Mit dieser Auffassung nicht vereinbar waren in der Folgezeit einzelne Kasuistiken, in denen neben Darier-typischer Morphen in umschriebenen Lokalisationen dann doch assoziierte Symptome beschrieben wurden, wie z.B. Unterbrechungen der Papillarlinien, Nagelveränderungen oder Keratosen der Handrücken und Schleimhäute $[13,16,19]$. Des Weiteren wurde darauf hingewiesen, dass epidermale Nävi typischerweise bereits in der frühen Kindheit auftreten, allenfalls ein zeitlich begrenztes Wachstum zeigen und ihre Ausprägung keinen Provokationsfaktoren unterliegt, z. B. einer Sonnenaggravation, wie dies für den Morbus Darier und auch für die lokalisierten Formen typisch ist [16]. Das lokalisierte Auftreten der DarierMorphen im Verlauf der Blaschko-Linien ist als Ausdruck einer Mosaikmanifestation des Morbus Darier gewertet worden $[19,20]$. Unter einem Mosaik versteht man einen Organismus, der aus mindestens zwei genetisch verschiedenen Zellklonen besteht, die sich aus einer einheitlichen Zygote entwickelt haben [21]. Im einfachsten Fall ist die Mosaikbildung auf eine postzygote Mutation zurückzuführen [22]. Das klinisch erkennbare Hervortreten der Blaschko-Linien hängt dabei vom Ausmaß der Mosaikbildung ab und ist offensichtlich häufiger als vermutet, da die in einzelnen Kasuistiken als zosteriform beschriebenen Manifestationen tatsächlich dem Verteilungsmuster der BlaschkoLinien zugeordnet werden können $[10,23]$. Darüber hinaus erklärt die Mosaikbildung auch das Auftreten assoziierter Symptome bei den lokalisierten Formen des Morbus Darier. Das Ausmaß der klinischen Manifestation der Dermatose hängt dabei vom Zeitpunkt der Mutation ab, die zur Mosaikbildung führt. Je früher die Mutation in der Embryogenese auftritt, desto ausgedehnter wird das klinische Bild [1]. Der Zeitpunkt der Mutation ist verantwortlich für das Ausmaß der lokalisierten Formen und für 
das Auftreten der assoziierten Symptome: Bei einer spät auftretenden Mutation werden die Darier-Morphen nur in einer sehr umschriebenen Lokalisation vorliegen, während assoziierte Symptome vollständig fehlen [19].

Auf Grund genetischer Befunde wurde der Mosaikbegriff erstmals von Happle in die Nävusdefinition aufgenommen. Demzufolge sind Nävi sichtbare, umschriebene, langfristig bestehende Haut- oder Schleimhautveränderungen, die ein genetisches Mosaik widerspiegeln [24]. Dabei ist die Mosaiknatur der Nävi nicht nur für epidermale Formen, sondern auch für kongenitale melanozytäre Nävi nachgewiesen worden [25]. In der Diskussion um einen sich verändernden Nävusbegriff hat Jung auf die Schnyder'sche Regel zur Erblichkeit von Hauttumoren hingewiesen $[21,27]$. Bei bestimmten kutanen Tumorformen, z. B. Trichoepitheliome oder Zylindrome, sind die multiplen disseminiert auftretenden Exemplare genetisch determiniert und werden autosomal-dominant vererbt, während die histogenetisch identischen singulären Exemplare keine Erblichkeit aufweisen. Im Gegensatz zu den singulär auftretenden Tumoren sind die multiplen Formen selten, entwickeln sich in frühen Lebensabschnitten und können assoziierte Symptome aufweisen.

Auch die segmentär angeordneten, nicht familiär zu beobachtenden, auf einer somit postzygoten Mutation beruhenden Tumorformen wurden von Jung der Gesetzmäßigkeit der Schnyderschen Regel unterstellt. Das Gesagte gilt z.B. für die Abgrenzung des Morbus Recklinghausen von segmentär auftretenden Varianten oder einzelnen Neurofibromen. Neben der Anwendung der Schnyderschen Regel bei bestimmten Tumorformen der Haut hat Jung die Gültigkeit der Regel auch auf den Morbus Darier und seine lokalisierten Formen übertragen [26]. In Ergänzung zur Nävusdefinition von Happle hat Jung folgerichtig darauf hingewiesen, dass sich der Mosaikzustand eines Nävus oft in einer segmentären Anordnung im Verlauf der Blaschko-Linien manifestiert [27]. Vor dem Hintergrund der von Happle und Jung geprägten Diskussion über einen modernen Nävusbegriff ist die Frage, ob die lokalisierten Formen des Morbus Darier als Nävus oder als Variante der klassischen Form der Dermatose zu werten sind, hinfällig, da beide Einschätzungen von einer genetischen Mosaikbildung ausgehen und sich somit gegenseitig keineswegs ausschließen [22].

Der hier vorgestellte Fall erfüllt die typischen Zeichen der lokalisierten Formen eines Morbus Darier. Neben einer unauffälligen Familienanamnese, dem Fehlen assoziierter Symptome und der Manifestation in einem hohen Lebensalter ist die außerordentlich umschriebene Manifestation der Dermatose zweifellos bemerkenswert. Die aufgrund des histopathologischen Befundes einer fokalen akantholytischen Dyskeratose zu diskutierenden Differenzialdiagnosen, z. B. eines warzigen Dyskeratoms, eines Morbus Grover oder einer papulösen akantholytischen Dyskeratose, konnten anhand der unterschiedlichen klinischen Befunde der jeweiligen Dermatosen sicher ausgeschlossen werden [28 - 30].

\section{Literatur}

1 Jung EG. Der segmentäre Morbus Darier. Akt Dermatol 2000; 26: 325 329

${ }^{2}$ Craddock N, Dawson E, Burge $S$ et al. The gene for Darier's disease maps to chromosome 12 q 23 - q 24.1. Hum Mol Genet 1993; 2: 1941 1943

${ }^{3}$ Sakuntabhai A, Ruiz-Perez V, Carter S et al. Mutations in APT2A2, encoding a Ca ${ }^{2+}$ pump, cause Darier disease. Nat Genet 1999; 21 : 252 253

${ }^{4}$ Burge SM, Wilkinson JD. Darier-White disease: A review of the clinical features in 163 patients. J Am Acad Dermatol 1992; 27: 40-50

${ }^{5}$ Nasemann T. Papulöse Krankheiten der Haut. In: Korting GW (Hrsg). Dermatologie in Praxis und Klinik. Stuttgart: Thieme, 1980

${ }^{6}$ Schanne R, Burg G, Braun-Falco O. Zur nosologischen Beziehung der Dyskeratosis follicularis (Darier) und des Pemphigus benignus chronicus familiaris (Hailey-Hailey). Hautarzt 1985; 36: 504-508

7 Jorda D, Revert A, Montesinos E et al. Unilateral Darier's disease. Int J Dermatol 1996; 35: $288-289$

${ }^{8}$ Niebauer G, Raff M. Morbus Darier in linearer Anordnung. Derm Mschr 1970; 156: 1047-1053

9 Perret C, Nödl F, Zaun H. Passagerer naeviformer Morbus Darier - eine Paraneoplasie? In: Gollnick H, Stadler R (Hrsg). Dia-Klinik 17. Weltkongress für Dermatologie. Stuttgart: Schattauer, 1987

${ }^{10}$ Scuppli R. Morbus Darier zosteriformis. Dermatologica 1962; 124: $274-275$

${ }^{11}$ Kellum RE, Haserick JR. Localized linear Keratosis follicularis. Arch Dermatol 1962; 86: 450-454

12 O'Malley M, Haake A, Goldsmith L et al. Localized Darier Disease. Arch Dermatol 1997; 133: 1134-1138

${ }^{13}$ Esche C, Pier A, Zumdick M et al. Morbus Darier im Verlauf der Blaschko-Linien. H + G 1995; 70: 758 - 760

${ }^{14}$ Svendsen IB, Albrectsen B. The prevalence of dyskeratosis follicularis (Darier's disease) in Denmark: an investigation of the heredity in 22 families. Acta Derm Venereol 1959; 39: 256 - 269

15 Wilkinson JD, Marsden RA, Dawber RPR. Review of Darier's disease in the Oxford region. Br J Dermatol 1977; Suppl 15: 15-16

${ }^{16}$ Munro CS, Cox NH. An acantholytic dyskeratotic epidermal naevus with other features of Darier's disease on the same side of the body. Br J Dermatol 1992; 127: 168 - 171

17 Starink TM, Woerdeman MJ. Unilateral systematized keratosis follicularis. A variant of Darier's disease or an epidermal naevus (acantholytic dyskeratotic epidermal naevus)? Br J Dermatol 1981; 105: 207 214

${ }^{18}$ Leeming JAL. Acquired linear naevus showing histological features of Keratosis follicularis. Br J Dermatol 1969; 81: 128-131

${ }^{19}$ Cambiaghi S, Brusasco A, Grimalt R et al. Acantholytic dyskeratotic epidermal nevus as a mosaic form of Darier's disease. J Am Acad Dermatol 1995; 32: $284-286$

${ }^{20}$ Jackson R. The lines of Blaschko: a review and reconsideration. $\mathrm{Br} \mathrm{J}$ Dermatol 1976; 95: 349-360

${ }^{21}$ Bolognia JL, Orlow SJ, Glick SA. Lines of Blaschko. J Am Acad Dermatol 1994; 31: 157 - 190

22 Happle R. Nichtmelanozytäre Nävi und nävoide Dermatosen. In: Traupe H, Hamm H (Hrsg). Pädiatrische Dermatologie. Berlin: Springer, 1999

${ }^{23}$ Wegen-Keijser v d MH, Prevoo RL, Bruynzeel DP. Acantholytic dyskeratotic epidermal naevus in a patient with guttate psoriasis on PUVA therapy. Br J Dermatol 1991; 124: 603-605

${ }^{24}$ Happle R. What is a nevus? A proposed definition of a common medical term. Dermatology 1995; 191: 1 - 5

${ }^{25}$ Robinson WA, Norris D, Elefanty A et al. Human acquired nevi are clonal. J Invest Dermatol 1996; 106: 883

26 Jung EG. Die „Schnydersche Regel“ zur Erblichkeit von Hauttumoren und ihre Ergänzung durch segmental angeordnete Fälle. Hautarzt 1989; 40: $761-762$

27 Jung EG. Was ist ein Naevus? Akt Dermatol 1999; 25: 60-65

28 Ackerman AB. Focal acantholytic dyskeratosis. Arch Dermatol 1972; 106: $702-706$

${ }^{29}$ Almeida de HL, Wolter M, Castro LAS et al. Papulöse akantholytische Dyskeratose. Hautarzt 2001; 52: $1101-1103$

${ }^{30}$ Metz J, Schröpl F. Zur Nosologie des Dyskeratoma segregans („Warty dyskeratoma“). Arch klin exp Derm 1970; 238: 21-37 